\title{
Die FMH und integrierte Versorgungssysteme
}

\begin{abstract}
Integrierte Versorgungsnetze sind eine der Möglichkeiten, die in den laufenden Diskussionen über die Zukunft des Gesundheitssystems besonders viel Raum einnehmen. Und es zeichnen sich am politischen Horizont allmählich konkrete Entscheide $a b$.

Gewiss, für einige ist das zwar nur eine Alibiübung und für andere ein bequemer Slogan. Wir jedoch beurteilen diesen Prozess als überaus positiv: Bereits vor vier Jahren hat die FMH zu diesem Thema in ihren nach wie vor aktuellen «Thesen» [1] klar Stellung bezogen - und sie unterstützt diese Entwicklung mit Überzeugung und dezidiertem Engagement.
\end{abstract}

\section{«Die FMH unterstützt die Entwicklung von integrierten Versorgungsnetzen mit Überzeugung und dezidiertem Engagement»}

Nach unserer Auffassung stellen integrierte Versorgungsnetze ein effizientes Instrument für eine qualitativ hochstehende Medizin dar, die in der gesamten Behandlungskette auf den Patienten ausgerichtet ist. Wir haben damit zudem die Möglichkeit, Einfluss auf das Gesundheitssystem zu nehmen, in welchem wir aktiv sind, und uns um die Finanzierung zu kümmern, da für jedes Netzwerk ein Vertrag zwischen den Ärzten und den Versicherern ausgehandelt wird.

Die Delegiertenversammlung der FMH hat vor zwei Wochen eine interessante Diskussion über diese integrierten Versorgungsnetze geführt.

Der VSAO hat der Versammlung das «PGS-Modell» vorgestellt, für das er seit langem plädiert. Anschliessend informierte Ruth Humbel, Präsidentin der parlamentarischen Subkommission, über die Arbeiten und Resultate zum Gesetzesentwurf «Managed Care», und Nello Castelli erläuterte die entsprechende Haltung von santésuisse. An den Überlegungen der Delegierten beteiligten sich auch mehrere ManagedCare-Spezialisten aus der Deutsch- und Westschweiz.

Worum ging es dabei?

\section{«Managed Care» beruht auf der Motivation der beteiligten Patienten, Ärzte und Versicherer. Zwang hat dabei nichts zu suchen, da er nichts Positives bewirkt»}

Klar ist, dass der Risikoausgleich differenzierter auszugestalten ist, damit die integrierten Versorgungssysteme korrekt funktionieren können - nur so werden die chronisch kranken Patienten nicht diskriminiert, die am meisten Kosten verursachen und am meisten von «Managed Care» profitieren können. Doch die Verbesserung des Risikoausgleichs, die 2012 in Kraft treten wird, reicht bestimmt nicht aus: Wir müssen - das ging aus unserer Diskussion klar hervor - mit unseren kantonalen Gesellschaften weiterhin sachgerechte Modelle entwickeln und uns für deren Realisierung einsetzen.

Wichtig ist auch die freie Wahl bei der Einführung von integrierten Versorgungsnetzen - was in der Delegiertenversammlung ebenfalls deutlich wurde.

Es liegt auf der Hand, dass «Managed Care» auf der Motivation der beteiligten Patienten, Ärzte und Versicherer beruht. Zwang hat dabei nichts zu suchen, da er nichts Positives bewirkt, die Qualität von «Managed Care» beeinträchtigt und das Erreichen der Ziele verhindert.

Bei zwei Elementen, die gegenwärtig diskutiert werden, geht es um diese wichtige Wahlfreiheit: zum einen bei der Budgetverantwortung der Ärzte und zum anderen bei der freien Wahl für alle Partner zwischen «Managed Care» und dem herkömmlichen System.

Diese beiden Punkte sind ausserordentlich wichtig. Deshalb werden wir uns dafür einsetzen, dass unser Standpunkt verstanden und berücksichtigt wird.

Die Patienten müssen ihr Versorgungssystem wirklich frei wählen können. Selbstverständlich sind dabei für die Förderung des Systems finanzielle Anreize notwendig, doch diese müssen in einem angemessenen Rahmen bleiben. Denn Übertreibungen kämen für den finanziell weniger gut gestellten Teil der Bevölkerung einem Zwang gleich.

Auch die Ärzte müssen ihren Arbeitsrahmen frei wählen und entscheiden können, wie sie sich in das System integrieren. «Managed Care» ist eine gute Variante, ... doch es muss auch ein Leben ausserhalb von Managed Care möglich sein!

Somit ist eine Koexistenz des gegenwärtigen Systems und der integrierten Versorgungsnetze anzustreben. Im derzeitigen System muss der Kontrahierungszwang garantiert bleiben, sonst wird sich die Ärzteschaft - das ist mittlerweile bekannt - politisch vehement zur Wehr setzen.

In den Diskussionen über «Managed Care» sind sich die FMH und santésuisse über viele zentrale Punkte einig - insbesondere über die Wahlfreiheit für alle Beteiligten, einschliesslich der Versicherer. Aus dieser Übereinstimmung, die auch den Vertragszwang im gegenwärtigen System umfasst, resultierte ein gemeinsames Papier, das die FMH und santésuisse Anfang Jahr den eidgenössischen Räten vorlegten.

Die Budgetverantwortung der Ärzte in integrierten Versorgungsnetzen wird in den bestehenden Netzen in vielen Fällen umgesetzt. Doch es gibt keinen Grund, sie für obligatorisch zu erklären, wie es das Parlament möchte. Es ist wichtig, dass in der Schweiz alle Arten von Modellen entwickelt und getestet werden können. Es wäre völlig kontraproduktiv, die Versuche in diesem Bereich einzuschränken.

Die FMH hat somit zu diesem aktuellen Thema mehrere klare Standpunkte. Wir befinden uns jetzt zweifellos in einer guten Position, um unsere Ideen in den kommenden Debatten zum Ausdruck und zur Geltung zu bringen!

Dr. med. Jacques de Haller, Präsident der FMH

1 www.fmh.ch/files/pdf2/Thesenpapier_Managed_Care_D.pdf 